\title{
BMJ Open Effectiveness of balance training in patients with chronic ankle instability: protocol for a systematic review and meta-analysis
}

\author{
Ju Wang, ${ }^{1}$ Di Zhang (D) , ${ }^{2}$ Tianyu Zhao, ${ }^{2}$ Jiang Ma (D) , ${ }^{1}$ Song Jin (D) ${ }^{2}$
}

To cite: Wang J, Zhang D, Zhao $\mathrm{T}$, et al. Effectiveness of balance training in patients with chronic ankle instability: protocol for a systematic review and meta-analysis. BMJ Open 2021;11:e053755. doi:10.1136/ bmjopen-2021-053755

- Prepublication history and additional supplemental material for this paper are available online. To view these files, please visit the journal online (http://dx.doi.org/10.1136/ bmjopen-2021-053755).

JW and DZ contributed equally

Received 22 May 2021

Accepted 02 September 2021

Check for updates

(C) Author(s) (or their employer(s)) 2021. Re-use permitted under CC BY-NC. No commercial re-use. See rights and permissions. Published by BMJ.

${ }^{1}$ School of Health Preservation and Rehabilitation, Chengdu University of Traditional Chinese Medicine, Chengdu, Sichuan,

China

${ }^{2}$ Rehabilitation Department, Hospital of Chengdu University of Traditional Chinese Medicine, Chengdu, Sichuan, China

Correspondence to

Dr Song Jin;

j1049147000@163.com

\section{ABSTRACT}

Introduction Lateral ankle sprains are one of the most common musculoskeletal injuries. Up to $70 \%$ of individuals who sustain lateral ankle sprains develop chronic ankle instability (CAl). Balance training has been used in patients with CAl, but the evidence for its efficacy is inconsistent. This systematic review and meta-analysis aims to determine the short-term (end of the treatment period) and long-term (6 months after treatment) effectiveness of balance training for patients with CAI.

Methods and analysis We will search PubMed, EMBASE, the Cochrane Library, Ovid, EBSCO-host, Pedro, ClinicalKey, ScienceDirect, Springer, China National Knowledge Infrastructure, Technology Periodical Database (VIP), WanFang Data and China Biology Medicine for reports of randomised trials of balance training in patients with CAl, from inception to 10 ctober 2021. The language will be restricted to English and Chinese, and articles will be screened and collected by two reviewers independently. Dynamic balance and functional ankle instability are the primary outcomes of this study. Secondary outcomes include pain, ankle range of motion, ankle strength and health-related quality of life. Review Manager V.5.3 software will be used for meta-analysis, and stratification analysis will be conducted for study quality according to the Jadad score. Subgroup and sensitivity analyses will be conducted. Grading of Recommendations, Assessment, Development and Evaluation will be used to assess confidence in the cumulative evidence. The protocol follows the Cochrane Handbook for Preferred Reporting Items for Systematic Reviews and Meta-Analysis Protocols guidelines.

Ethics and dissemination Ethical approval is not required for literature-based studies. The results will be disseminated through peer-reviewed publications.

\section{INTRODUCTION}

Lateral ankle sprains are one of the most common musculoskeletal injuries. ${ }^{1}$ More than 2 million cases of ankle sprains occur each year in the USA. ${ }^{2}$ With the extension of the average life span, the incidence rate has gradually increased. ${ }^{3} \mathrm{Up}$ to $70 \%$ of patients may not receive proper rehabilitation after the initial injury, resulting in chronic ankle instability (CAI) ${ }^{45} \mathrm{CAI}$ is characterised by a
Strengths and limitations of this study

We will collect a broad range of outcomes to assess the potential benefits of balance training for patients with chronic ankle instability (CAl).

- This work could be helpful in verifying the shortterm and long-term efficacy of balance training in patients with CAl.

- We will conduct a stratification analysis according to study quality to make a reliable conclusion.

- Only randomised controlled trials (RCTs; not including quasi-RCTs) will be included in this analysis.

- A variety of control interventions and differences in sample size, time-course of treatment, and population may increase heterogeneity and bias.

history of one significant lateral ankle sprain, which manifested as sensory 'giving way' and instability, recurrent ankle sprain, joint laxity, pain, swelling and impaired ankle function. ${ }^{6}$ The residual symptoms last at least 1 year after the acute ankle sprain, ${ }^{7}$ affect the level of daily activity and quality of life, and possibly increase the risk of chronic diseases in patients with CAI. ${ }^{38}$

The factors leading to CAI are complex and may be related to motor perception disturbance and mechanoreceptor injury, ${ }^{9}$ and the loss of balance is a determinant factor. ${ }^{10-12}$ Freeman $e t a l^{13}$ found that afferent feedback changes are the main functional mechanisms of recurrent ankle sprains. Hertel ${ }^{14}$ suggests that joint ligament injury of the lateral ankle affects the feedback loop of proprioceptive sensation, resulting in sensorimotor disturbances. It manifests as defects in neuromuscular control, proprioception, posture control, strength and range of motion (ROM) ${ }^{15-18}$ Studies have shown that pain and swelling might negatively affect motor perception, resulting in decreased motor function and increased risk of reinjury and post-traumatic arthritis. ${ }^{19} 20$ 
Non-operative treatment is recommended as the primary treatment for patients with CAI. ${ }^{21}$ Balance training, the main component of rehabilitation plans, ${ }^{22}$ has been widely used for patients with CAI. ${ }^{23}$ Balance training can promote the recovery of motor perception by stimulating the receptors of the ankle joint capsule and ligaments, increasing the input of motor sensation and activating gamma motor neurons. ${ }^{24} 25$

Although two systematic reviews in $2008^{23}$ and $2017^{26}$ assessed balance training efficacy for patients with ankle sprains, the former focused on patients with acute ankle sprains only, and the latter compared patients with healthy controls. In addition, a systematic review published in $2021^{27}$ only scanned three databases and analysed the shortterm effects of balance training. However, several relative randomised controlled trials (RCTs) published after the final retrieval deadline of April 2021, ${ }^{28-31}$ and control interventions, such as whole-body vibration training and ROM training, have not been compared with balance training.

It is essential to investigate the duration of treatment effects of balance training in patients with CAI for clinicians in the decision-making process. ${ }^{32}$ Studies have defined 6 months post-intervention as a long-term effect assessment point for CAI. ${ }^{33}$ Studies used a follow-up period of 6-12 months to evaluate the long-term effects of CAI ${ }^{33-35}$ Wright and Linens ${ }^{33}$ reported that $42.9 \%$ of participants had improved sufficiently at 6-month follow-up, and no longer meet the criteria of CAI inclusion. ${ }^{6}$ Thus, it is an important follow-up point for clinical decisions. Therefore, we will define long-term effects as 6 months after treatment based on the compliance of subjects, clinical significance and the literature research findings. However, there is no systematic review on the subject to summarise its long-term effects. Thus, we expect to conduct a systematic review and meta-analysis to evaluate and synthesise the published RCTs and update a definite conclusion of the short-term (end of the treatment period) and long-term (6 months after treatment) efficacy of balance training for patients with CAI.

\section{METHODS AND ANALYSIS}

The protocol follows the Cochrane Handbook for the Preferred Reporting Items for Systematic Reviews. We used the Preferred Reporting Items for Systematic Reviews and Meta-Analysis Protocols checklist to guide the reporting of this protocol.

\section{Eligibility criteria}

Types of studies

The types of studies include RCTs on balance training for patients with CAI. A randomised study will be conducted if a trial mentions the phrase 'randomisation', and blinding is not restricted. The language of the RCTs will be limited to English and Chinese.

Types of participants

CAI was diagnosed according to the International Ankle Consortium. ${ }^{6}$
1. Patients with CAI (aged $\geq 18$ years) of any sex and ethnicity were enrolled.

2. Patients with a history of an ankle sprain that occurred at least 12 months prior to study enrolment.

3. Patients with a history of injured ankle joint 'giving way', and/or recurrent sprain, and/or 'feelings of instability', and with a Cumberland Ankle Instability Tool (CAIT) score of $\leq 24$.

\section{Types of interventions}

The treatment group includes balance training with or without conventional rehabilitation, while the control group includes any type of rehabilitation, except balance training.

\section{Main outcome(s)}

Primary outcomes

1. Dynamic stability will be evaluated using the Star Excursion Balance Test. It is an excellent functional index for monitoring changes in CAI after rehabilitation. $^{36}$

2. The functional ankle instability will be evaluated by the CAIT. It is a simple, reliable and valid questionnaire. ${ }^{37}$

\section{Secondary outcomes}

1. The pain intensity will be measured using the Visual Analogue Scale or Numerical Rating Scale.

2. The ankle ROM will be measured, including dorsiflexion, plantar flexion, inversion and eversion movements.

3. Ankle strength (inversion/eversion and dorsiflexion/ plantarflexion) will be measured.

4. Health-related quality of life will be measured by the 36-item Short-Form Health Survey.

\section{Timing and effect measures}

We will define short-term effects as the end of the treatment period and long-term effects as 6 months after treatment. This study will analyse the primary and secondary outcomes at the following time points: end of the treatment period (short-term) and 6 months after treatment (long-term).

\section{Exclusion criteria}

Case reports, animal mechanism studies, self-pre-control and post-control, expert experience, duplicated publications and quasi-RCTs will be excluded.

\section{Information sources and search strategy}

We will search PubMed, EMBASE, the Cochrane Library, Ovid, EBSCO-host, Pedro, ClinicalKey, ScienceDirect, Springer, China National Knowledge Infrastructure, Technology Periodical Database (VIP), WanFang Data and China Biology Medicine for reports of randomised trials of balance training in patients with CAI, from inception to 1 October 2021. Medical Subject Headings, text words, and word variants, such as "balance," "coordination," "proprioception," "equilibrium," "vestibular sense," "hop stabilization," "training," "chronic ankle instability," 


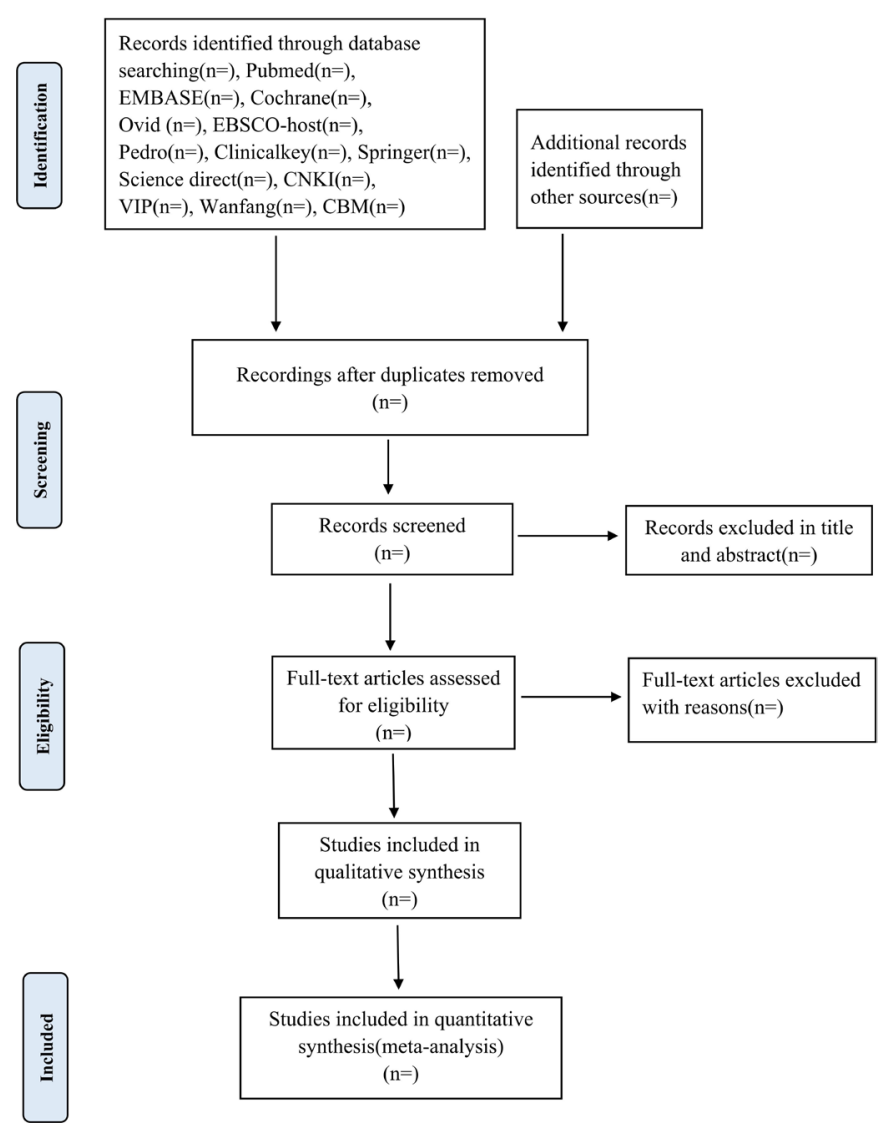

Figure 1 Screening process.

"ankle instability," "CAI," "ankle injury," "ankle sprain," "syndesmotic injury," will be searched for in all databases. The language will be restricted to English and Chinese. In addition, the reference lists of selected papers will be checked manually. Detailed search strategies for databases can be found in the online supplemental file.

\section{Data collection}

Two independent reviewers (JM and TZ) will scan the titles and abstracts according to the inclusion and exclusion criteria and screen articles using their full-text version. They will extract the data into a self-designed data extraction form and cross-check the selection results if they satisfy the criteria. Any disagreements will be arbitrated by a third reviewer $(\mathrm{SJ})$. The selection procedure is shown in figure 1 .

\section{Data extraction and management}

Two reviewers (JW and DZ) will extract data from the included studies independently. Multi-arm RCTs will be divided into two arms according to the intervention. Balance training or balance training combined with conventional rehabilitation will be considered the treatment group, and other interventions without balance training will be the control group. The data extraction form will include basic information (article title, first author, publication year, country, mean age of the population, and number of male and female patients), study design (RCT design, blinding method, randomisation, sample size), interventions (including the type, frequency, session, duration and follow-up time), outcome measures and conclusions. Diversity will be resolved by a third reviewer (SJ).

\section{Assessment of risk of bias and quality}

Two reviewers (JW and JM) will assess the risk of bias of the included trials using the Cochrane Collaboration's risk of bias assessment tool. We will evaluate the risk of bias (low, high and unclear risk) in six areas, including performance bias (blinding of participants and personnel), selection bias (random sequence generation, allocation concealment), detection bias (blinding of outcome assessment), attrition bias (incomplete outcome data), reporting bias (selective reporting) and other biases. We will conduct a stratification analysis for study quality according to the Jadad score. For duplicate publications, we will select only the original. The final decisions will be made by a third reviewer (SJ) if inconsistent results appear.

\section{Management of missing data}

For incomplete data or missing data, we will contact the corresponding author to request additional information. Otherwise, related studies will be excluded.

\section{Assessment of heterogeneity}

The Q-test and $\mathrm{I}^{2}$ statistic will be used to assess the heterogeneity of the included studies, as the criteria: $\mathrm{I}^{2}<50 \%$ indicates low heterogeneity, while $\mathrm{I}^{2} \geq 50 \%$ indicates high heterogeneity.

\section{Assessment of publication bias}

We will use the funnel plot to visually assess publication bias if the number of included trials is more than $10 .^{38}$

\section{Data analysis and synthesis}

Review Manager (V.5.3, Nordic Cochrane Centre, Copenhagen, Denmark) will be used. The relative risk will be used to analyse dichotomous risk. The fixed-effects model will be used to combine the data if the statistical heterogeneity is low $\left(\mathrm{I}^{2}<50 \%\right)$, or a random-effects model will be used if the statistical heterogeneity is high $\left(\mathrm{I}^{2} \geq 50 \%\right)$. The mean difference (MD) with $95 \%$ CI will be used for the continuous variables, and standardised MD and $95 \%$ CI will be used for the continuous variables if the units are different. When quantitative evaluation is not available, we will provide a qualitative description of the individual study results.

\section{Subgroup analysis and investigation of heterogeneity}

We will use subgroup analysis to explore the causes of heterogeneity according to the following potential factors: control group types (whole-body vibration training, ROM training and strength training).

\section{Sensitivity analysis}

Sensitivity analysis will be conducted according to the age of patients, time of initiation and course of treatment, intervention method and sample size. 


\section{Grading the quality of evidence}

Two reviewers will independently assess the quality of evidence for outcomes using the Grading of Recommendations, Assessment, Development and Evaluation guidelines. ${ }^{39}$ The strength of the body of evidence is divided into four levels: high, medium, low and very low. ${ }^{40}$

\section{Patient and public involvement}

Patients and/or the public are not involved in the design, conduct, reporting or dissemination plans of this research.

\section{Ethics and dissemination}

Ethical approval was not required in this study because no data are related to an individual patient. The results will be disseminated through peer-reviewed publications.

\section{DISCUSSION}

Several studies revealed that previous injury, balance deficit, pain, decline of eccentric muscle strength and loss of ankle dorsiflexion are related to a recurrent ankle sprain in patients with CAI. ${ }^{41-43}$ Donovan and Hertel ${ }^{44}$ presented a new paradigm rehabilitation for patients with CAI within four broad domains, namely functional activities, ROM, strength and balance. The rehabilitation plan aims to improve the related functional defects, reduce pain, prevent recurrent ankle sprains and improve patients' quality of life. ${ }^{2145}{ }^{46}$ Clinical reports have shown that balance training effectively treats patients with CAI. ${ }^{24}{ }^{25}{ }^{47-51}$ Though several systematic reviews evaluated the efficacy of balance training for CAI, the conclusion is indefinite for various limitations of methods. ${ }^{232627}$ Thus, to better understand the potential benefits of balance training and conduct clinical decision-making for CAI, we will design this study to determine the short-term and long-term efficacy of balance training for CAI.

In this review, stratification analysis will be carried out for the study quality based on the Jadad score (highquality study (Jadad score $\geq 4$ ) and low-quality study (Jadad score $\leq 3)$ ). ${ }^{52}$ Subgroup analysis will be considered according to interventions of control groups, time/ course of treatment and age (if heterogeneity is high $\left.\left(\mathrm{I}^{2} \geq 50 \%\right)\right)$. Potential limitations may affect the results, such as the variety of control interventions and differentiation in sample size, time/course of treatment and population. New unpublished data may not be available at the time of submission of this review.

Above all, various follow-up outcomes need to be evaluated in future studies to better understand the potential benefits of balance training and provide evidence-based decisions for clinicians and researchers regarding CAI.

Contributors JW and DZ made equal contributions to this study. JW conceived the study and developed the first frame of this manuscript. JW and DZ drafted the manuscript and contributed to the development of the search strategy. JM and TZ will read the full texts of the studies and extract the data. JW and DZ will perform the data synthesis. SJ will arbitrate in cases of any disagreement. The manuscript was revised by SJ. All authors read and approved the final manuscript.
Funding This study was supported by the Department of Science and Technology of Sichuan Province (Grant No.2020YFS0381).

Competing interests None declared.

Patient consent for publication Not applicable.

Provenance and peer review Not commissioned; externally peer reviewed.

Supplemental material This content has been supplied by the author(s). It has not been vetted by BMJ Publishing Group Limited (BMJ) and may not have been peer-reviewed. Any opinions or recommendations discussed are solely those of the author(s) and are not endorsed by BMJ. BMJ disclaims all liability and responsibility arising from any reliance placed on the content. Where the content includes any translated material, BMJ does not warrant the accuracy and reliability of the translations (including but not limited to local regulations, clinical guidelines, terminology, drug names and drug dosages), and is not responsible for any error and/or omissions arising from translation and adaptation or otherwise.

Open access This is an open access article distributed in accordance with the Creative Commons Attribution Non Commercial (CC BY-NC 4.0) license, which permits others to distribute, remix, adapt, build upon this work non-commercially, and license their derivative works on different terms, provided the original work is properly cited, appropriate credit is given, any changes made indicated, and the use is non-commercial. See: http://creativecommons.org/licenses/by-nc/4.0/.

\section{ORCID iDs}

Di Zhang http://orcid.org/0000-0001-6761-0741

Jiang Ma http://orcid.org/0000-0002-7059-062X

Song Jin http://orcid.org/0000-0003-4725-780X

\section{REFERENCES}

1 Waterman BR, Belmont PJ, Cameron KL, et al. Epidemiology of ankle sprain at the United States military Academy. Am J Sports Med 2010;38:797-803.

2 Waterman BR, Owens BD, Davey S, et al. The epidemiology of ankle sprains in the United States. J Bone Joint Surg Am 2010;92:2279-84.

3 Hiller CE, Nightingale EJ, Raymond J, et al. Prevalence and impact of chronic musculoskeletal ankle disorders in the community. Arch Phys Med Rehabil 2012;93:1801-7.

4 Gribble PA, Bleakley CM, Caulfield BM, et al. Evidence review for the 2016 international ankle Consortium consensus statement on the prevalence, impact and long-term consequences of lateral ankle sprains. Br J Sports Med 2016;50:1496-505.

5 Coughlin MJ, Schenck RC, Grebing BR, et al. Comprehensive reconstruction of the lateral ankle for chronic instability using a free gracilis graft. Foot Ankle Int 2004;25:231-41.

6 Gribble PA, Delahunt E, Bleakley C, et al. Selection criteria for patients with chronic ankle instability in controlled research: a position statement of the International ankle Consortium. $J$ Orthop Sports Phys Ther 2013;43:585-91.

7 Vallandingham RA, Gaven SL, Powden CJ. Changes in Dorsiflexion and dynamic postural control after Mobilizations in individuals with chronic ankle instability: a systematic review and meta-analysis. $J$ Athl Train 2019;54:403-17.

8 Beynnon BD, Murphy DF, Alosa DM. Predictive factors for lateral ankle Sprains: a literature review. J Athl Train 2002;37:376-80.

9 Thompson C, Schabrun S, Romero R, et al. Factors contributing to chronic ankle instability: a systematic review and meta-analysis of systematic reviews. Sports Med 2018;48:189-205.

10 Arnold BL, De La Motte S, Linens S, et al. Ankle instability is associated with balance impairments: a meta-analysis. Med Sci Sports Exerc 2009;41:1048-62.

11 Hiller CE, Nightingale EJ, Lin C-WC, et al. Characteristics of people with recurrent ankle sprains: a systematic review with meta-analysis. Br J Sports Med 2011;45:660-72.

12 Munn J, Sullivan SJ, Schneiders AG. Evidence of sensorimotor deficits in functional ankle instability: a systematic review with metaanalysis. J Sci Med Sport 2010;13:2-12.

13 Freeman MA, Dean MR, Hanham IW. The etiology and prevention of functional instability of the foot. $J$ Bone Joint Surg Br 1965;47:678-85.

14 Hertel J. Sensorimotor deficits with ankle sprains and chronic ankle instability. Clin Sports Med 2008;27:353-70.

15 Willems TM, Witvrouw E, Delbaere K, et al. Intrinsic risk factors for inversion ankle sprains in male subjects: a prospective study. $\mathrm{Am} J$ Sports Med 2005;33:415-23. 
16 Willems TM, Witvrouw E, Delbaere K, et al. Intrinsic risk factors for inversion ankle sprains in females--a prospective study. Scand $J$ Med Sci Sports 2005;15:336-45.

17 David P, Halimi M, Mora I, et al. Isokinetic testing of evertor and invertor muscles in patients with chronic ankle instability. $J$ Appl Biomech 2013;29:696-704.

18 Hertel J. Functional anatomy, Pathomechanics, and pathophysiology of lateral ankle instability. J Ath/ Train 2002;37:364-75.

19 Delahunt E, Bleakley CM, Bossard DS, et al. Clinical assessment of acute lateral ankle sprain injuries (roast): 2019 consensus statement and recommendations of the International ankle Consortium. $\mathrm{Br} \mathrm{J}$ Sports Med 2018;52:1304-10.

20 Xue Xiao'ao, Ma T, Li Q, et al. Chronic ankle instability is associated with proprioception deficits: a systematic review and meta-analysis. J Sport Health Sci 2021;10:182-91.

21 Doherty C, Bleakley C, Delahunt E, et al. Treatment and prevention of acute and recurrent ankle sprain: an overview of systematic reviews with meta-analysis. Br J Sports Med 2017;51:113-25.

22 Tsikopoulos K, Mavridis D, Georgiannos D, et al. Efficacy of nonsurgical interventions on dynamic balance in patients with ankle instability: a network meta-analysis. J Sci Med Sport 2018;21:873-9.

23 McKeon PO, Hertel J. Systematic review of postural control and lateral ankle instability. part II: is balance training clinically effective? J Athl Train 2008;43:305-15.

24 Cruz-Diaz D, Lomas-Vega R, Osuna-Pérez MC, et al. Effects of 6 weeks of balance training on chronic ankle instability in athletes: a randomized controlled trial. Int J Sports Med 2015;36:754-60.

25 Burcal CJ, Sandrey MA, Hubbard-Turner T, et al. Predicting dynamic balance improvements following 4-weeks of balance training in chronic ankle instability patients. J Sci Med Sport 2019;22:538-43.

26 Kosik KB, McCann RS, Terada M, et al. Therapeutic interventions for improving self-reported function in patients with chronic ankle instability: a systematic review. Br J Sports Med 2017;51:105-12.

27 Mollà-Casanova S, Inglés M, Serra-Añó P. Effects of balance training on functionality, ankle instability, and dynamic balance outcomes in people with chronic ankle instability: systematic review and meta-analysis. Clin Rehabil 2021;2692155211022009:2692155 211022009.

28 Kim K-M, Estudillo-Martínez MD, Castellote-Caballero Y, et al. ShortTerm effects of balance training with Stroboscopic vision for patients with chronic ankle instability: a single-blinded randomized controlled trial. Int J Environ Res Public Health 2021;18:5364.

29 Chang W-D, Chen S, Tsou Y-A. Effects of whole-body vibration and balance training on female athletes with chronic ankle instability. $J$ Clin Med 2021;10:5364:2380.

30 Uzlașır S, Özdıraz KY, Dağ O, et al. The effects of stroboscopic balance training on cortical activities in athletes with chronic ankle instability. Phys Ther Sport 2021;50:50-8.

31 VanDeMark LH, Vander Vegt CB, Ford CB, et al. Progressive visual occlusion and postural control responses in individuals with and without chronic ankle instability. J Sport Rehabil 2021:1-6.

32 Tsikopoulos K, Mavridis D, Georgiannos D, et al. Does multimodal rehabilitation for ankle instability improve patients' Self-assessed functional outcomes? A network meta-analysis. Clin Orthop Relat Res 2018;476:1295-310.

33 Wright CJ, Linens SW. Patient-Reported efficacy 6 months after a 4-week rehabilitation intervention in individuals with chronic ankle instability. J Sport Rehabil 2017;26:250-6.
34 Cruz-Díaz D, Lomas Vega R, Osuna-Pérez MC, et al. Effects of joint mobilization on chronic ankle instability: a randomized controlled trial. Disabil Rehabil 2015;37:601-10.

35 Eils E, Rosenbaum D. A multi-station proprioceptive exercise program in patients with ankle instability. Med Sci Sports Exerc 2001;33:1991-8.

36 Hale SA, Hertel J, Olmsted-Kramer LC. The effect of a 4-week comprehensive rehabilitation program on postural control and lower extremity function in individuals with chronic ankle instability. $J$ Orthop Sports Phys Ther 2007;37:303-11.

37 Hiller CE, Refshauge KM, Bundy AC, et al. The Cumberland ankle instability tool: a report of validity and reliability testing. Arch Phys Med Rehabil 2006;87:1235-41.

38 Rothstein HR, Sutton AJ, Borenstein M. Publication bias and metaanalysis: prevention, assessments and adjustments 2005.

39 Guyatt GH, Oxman AD, Vist GE, et al. Grade: an emerging consensus on rating quality of evidence and strength of recommendations. BMJ 2008;336:924-6.

40 Guyatt GH, Oxman AD, Schünemann HJ, et al. Grade guidelines: a new series of articles in the Journal of clinical epidemiology. J Clin Epidemiol 2011;64:380-2.

41 Abdel-aziem AA, Draz AH. Chronic ankle instability alters eccentric eversion/inversion and dorsiflexion/plantarflexion ratio. J Back Musculoskelet Rehabil 2014;27:47-53.

42 Alghadir $\mathrm{AH}$, lqbal ZA, lqbal $\mathrm{A}$, et al. Effect of chronic ankle sprain on pain, range of motion, proprioception, and balance among athletes. Int J Environ Res Public Health 2020;17. doi:10.3390/ ijerph17155318. [Epub ahead of print: 2307 2020].

43 Brown C, Ross S, Mynark R. Assessing functional ankle instability with joint position sense, time to stabilization, and electromyography. J Sport Rehabil 2004;13:122-34.

44 Donovan L, Hertel J. A new paradigm for rehabilitation of patients with chronic ankle instability. Phys Sportsmed 2012;40:41-51.

45 Al Adal S, Pourkazemi F, Mackey M, et al. The prevalence of pain in people with chronic ankle instability: a systematic review. J Athl Train 2019;54:662-70.

46 Powden CJ, Hoch JM, Hoch MC. Rehabilitation and improvement of health-related quality-of-life Detriments in individuals with chronic ankle instability: a meta-analysis. J Athl Train 2017;52:753-65.

47 Youssef NM, Abdelmohsen AM, Ashour AA, et al. Effect of different balance training programs on postural control in chronic ankle instability: a randomized controlled trial. Acta Bioeng Biomech 2018;20:159-69.

48 McKeon PO, Ingersoll CD, Kerrigan DC, et al. Balance training improves function and postural control in those with chronic ankle instability. Med Sci Sports Exerc 2008;40:1810-9.

49 Anguish B, Sandrey MA. Two 4-week Balance-Training programs for chronic ankle instability. J Athl Train 2018;53:662-71.

50 Sierra-Guzmán R, Jiménez-Diaz F, Ramírez C, et al. Whole-BodyVibration training and balance in recreational athletes with chronic ankle instability. J Athl Train 2018;53:355-63.

51 Minoonejad H, Karimizadeh Ardakani M, Rajabi R, et al. Hop stabilization training improves neuromuscular control in college Basketball players with chronic ankle instability: a randomized controlled trial. J Sport Rehabil 2019;28:576-83.

52 Jadad AR, Moore RA, Carroll D, et al. Assessing the quality of reports of randomized clinical trials: is blinding necessary? Control Clin Trials 1996;17:1-12. 